九州大学学術情報リポジトリ

Kyushu University Institutional Repository

\title{
PAIRWISE BALANCED, SEMI-REGULAR AND REGULAR GROUP DIVISIBLE DESIGNS
}

Sinha, Kishore

Department of Statistics, Birsa Agricultural University

Kageyama, Sanpe i

Department of Mathematics, Hiroshima University

https://doi.org/10.5109/13376

出版情報: Bulletin of informatics and cybernetics. 22 (1/2), pp.55-57, 1986-03. Research Association of Statistical Sciences

バージョン :

権利関係 : 


\title{
PAIRWISE BALANCED, SEMI-REGULAR AND REGULAR GROUP DIVISIBLE DESIGNS
}

\author{
By
}

\section{Kishore SinHA* and Sanpei KAGEYAMA**}

\begin{abstract}
From a regular group divisible design a method of constructing a pairwise balanced design and a semi-regular group divisible design is given.
\end{abstract}

\section{Introduction}

Sinha and Nigam [6] obtained balanced arrays and main-effect plans from regular group divisible designs. Here a method of constructing pairwise balanced designs and semi-regular group divisible designs from regular group divisible designs is given. The reference to design numbers are due to Clatworthy [2]. For definitions of some technical terms in this paper, refer to Raghavarao [5].

\section{Statements} follows.

We present one construction method of semi-regular group divisible designs as

THEOREM 1. The existence of a regular group divisible design with parameters

(1) $v=m n=b, r=m-1=k, \lambda_{1}=0, \lambda_{2}=1 ; m, n=m-2$

implies the existence of a semi-regular group divisible design with parameters

(2) $v=m(n+1), b=m n+1, r=m-1, k=m, \lambda_{1}=0, \lambda_{2}=1 ; m^{*}=m, n^{*}=m-1$

and a pairwise balanced design with parameters

(3) $v=m(n+1), b=m(n+1)+1, r=m, k_{i}=m$ or $m-1, \lambda=1$.

PROOF. Let the $m n$ treatments in the original regular group divisible design be arranged in an $n \times m$ array (i.e., $m$ groups of $n$ treatments each) as:

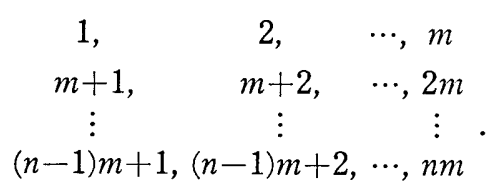

* Department of Statistics, Birsa Agricultural University, Ranchi 834006, India

** Department of Mathematics, Hiroshima University, Hiroshima 734, Japan 
Any two treatments in the same group $\{i, m+i, \cdots,(n-1) m+i\}(i=1,2, \cdots, m)$ are first associates, otherwise they are second associates. Now, to each block of a regular group divisible design with parameters (1), we add a treatment $(n m+i)$ if there is no treatment from the $i$-th group $(i=1,2, \cdots, m)$, and further add a new block $(n m+1$, $n m+2, \cdots, n m+m)$ consisting of " $m$ " new treatments to this arrangment. Thus, we get a semi-regular group divisible design with parameters (2) whose $m(n+1)$ treatments can now be arranged in an $(n+1) \times m$ array. Next, to the above semi-regular group divisible design with parameters (2), add $m$ blocks each of size $(n+1)$ consisting of elements in $m$ columns of the $(n+1) \times m$ array. Then we get a pairwise balanced design with parameters (3). Thus, the proof is completed.

REMARK: The existence of a regular group divisible design with parameters (1) is equivalent to the existence of a finite affine plane of order $n+1$. For, Theorem 8.6.2 in Raghavarao [5] gives the proof of Necessity of the result, while as Sufficiency part a reverse construction process is similarly provided. Thus, as a starting design in Theorem 1 , one can choose a finite affine plane also.

\section{Illustration}

(i) Semi-regular group divisible design: Let us consider a regular group divisible design, R54, with parameters $v=8=b, r=3=k, \lambda_{1}=0, \lambda_{2}=1, m=4, n=2$, which yields a semi-regular group divisible design, SR41, with parameters $v=12, b=9, r=3, k=4, \lambda_{1}=0$, $\lambda_{2}=1, m=4, n=3$, having blocks, $(1,2,4,11),(2,3,5,12),(3,4,6,9),(4,5,7,10),(5,6$, $8,11),(1,6,7,12),(2,7,8,9),(1,3,8,10),(9,10,11,12)$. The $3 \times 4$ array is

$\begin{array}{rrrr}1 & 2 & 3 & 4 \\ 5 & 6 & 7 & 8 . \\ 9 & 10 & 11 & 12\end{array}$

(ii) Pairwise balanced design: By adding the following four blocks, (1, 5, 9), (2, $6,10),(3,7,11),(4,8,12)$, consisting of elements in the 4 columns of the above array to the above-constructed semi-regular group divisible design (i), one can obtain a pairwise balanced design with parameters $v=12, b=13, r=4, k_{i}=3$ or $4, \lambda=1$.

\section{Some Semi-Regular Designs}

Now corresponding to the regular group divisible designs, $\mathrm{R}-54,114,153,183,191$, 202 in Clatworthy [2], we can obtain semi-regular group divisible designs with parameters as in SR-41, 58, 75, 96, 104, 110, respectively. The references to design numbers only indicate that a solution to each of these semi-regular group divisible designs is known in Clatworthy [2], but it can be checked that designs corresponding to SR $-41,58,75$ and 96 constructed here are also isomorphic to designs in Clatworthy [2]. The last two designs may yield non-isomorphic solutions to SR-104 and 110 in Clatworthy [2]. Corresponding to an unknown regular group divisible design with parameters

$$
\mathrm{R}-\mathrm{X}: v=99=b, r=10=k, \lambda_{1}=0, \lambda_{2}=1, m=11, n=9 \text {, }
$$


there is possibility of getting an unknown semi-regular group divisible design with parameters

$$
\text { SR }-\mathrm{X}: v=110, b=100, r=10, k=11, \lambda_{1}=0, \lambda_{2}=1, m=11, n=10 .
$$

The existence of the design $\mathrm{R}-\mathrm{X}$ is unknown in the sense that this is not found in Clatworthy [2], Freeman [3], Kageyama and Tanaka [4] and other available papers; and also their existence is not ruled out by the non-existence theorem for such symmetric designs in Bose and Connor [1]. Incidentally, the following two regular group divisible designs are also unknown within the scope of $r, k \leqq 10$ :

$$
\begin{aligned}
& v=b=45, r=k=7, \lambda_{1}=0, \lambda_{2}=1 ; m=15, n=3, \\
& v=b=75, r=k=9, \lambda_{1}=0, \lambda_{2}=1 ; m=25, n=3 .
\end{aligned}
$$

\section{Some Pairwise Balanced Designs}

Corresponding to the regular group divisible designs, $\mathrm{R}-54,114,153,183,191,202$, $\mathrm{X}$, we will obtain the following pairwise balanced designs, respectively.

$$
\begin{aligned}
& \text { (i) } v=12, b=13, r=4, k_{1}=4, k_{2}=3, \lambda=1, \\
& \text { (ii) } v=20, b=21, r=5, k_{1}=5, k_{2}=4, \lambda=1, \\
& \text { (iii) } v=30, b=31, r=6, k_{1}=6, k_{2}=5, \lambda=1, \\
& \text { (iv) } v=56, b=57, r=8, k_{1}=8, k_{2}=7, \lambda=1, \\
& \text { (v) } v=72, b=73, r=9, k_{1}=9, k_{2}=8, \lambda=1, \\
& \text { (vi) } v=90, b=91, r=10, k_{1}=10, k_{2}=9, \lambda=1, \\
& \text { (vii) } v=110, b=111, r=11, k_{1}=11, k_{2}=10, \lambda=1 .
\end{aligned}
$$

The existence of the last design (vii) is unknown.

\section{References}

[1] Bose, R.C. and CONNOR, W.S.: Combinatorial properties of group divisible incomplete block designs, Ann. Math. Statist. 23 (1952), 367-383.

[2] Clatworthy, W.H.: Tables of Two-Associate-Class Partially Balanced Designs, National Bureau of Standards, Applied Mathematics 63, Washington, D.C., 1973.

[3] FREEMAN, G.H.: A cyclic method of constructing regular group divisible incomplete block designs, Biometrika 63 (1976), 555-558.

[4] Kageyama, S. and Tanaka, T.: Some families of group divisible designs, J. Statist. Plann. Inf. 5 (1981), 231-241.

[5] Raghavarao, D.: Constructions and Combinatorial Problems in Design of Experiments, John Wiley, New York, 1971.

[6] Sinha, K. and Nigam, A.K. Balanced arrays and main-effect plans from regular group divisible designs, J. Statist. Plann. Inf. 8 (1983), 223-229.

Communicated by Ch. Asano

Received November 22, 1984 